\title{
A mechanistic role for cardiac myocyte apoptosis in heart failure
}

\author{
Detlef Wencker, ${ }^{1,2}$ Madhulika Chandra, ${ }^{1}$ Khanh Nguyen, ${ }^{1,2}$ Wenfeng Miao, ${ }^{1}$ \\ Stavros Garantziotis, ${ }^{1}$ Stephen M. Factor, ${ }^{1,3}$ Jamshid Shirani, ${ }^{1,3}$ Robert C. Armstrong, ${ }^{4}$ \\ and Richard N. Kitsis ${ }^{1,2}$
}

\author{
${ }^{1}$ Department of Medicine (Molecular Cardiology), \\ ${ }^{2}$ Department of Cell Biology, and \\ ${ }^{3}$ Department of Pathology, Albert Einstein College of Medicine, Bronx, New York, USA \\ ${ }^{4}$ Idun Pharmaceuticals, San Diego, California, USA
}

\begin{abstract}
Heart failure is a common, lethal condition whose pathogenesis is poorly understood. Recent studies have identified low levels of myocyte apoptosis (80-250 myocytes per $10^{5}$ nuclei) in failing human hearts. It remains unclear, however, whether this cell death is a coincidental finding, a protective process, or a causal component in pathogenesis. Using transgenic mice that express a conditionally active caspase exclusively in the myocardium, we demonstrate that very low levels of myocyte apoptosis (23 myocytes per $10^{5}$ nuclei, compared with 1.5 myocytes per $10^{5}$ nuclei in controls) are sufficient to cause a lethal, dilated cardiomyopathy. Interestingly, these levels are four- to tenfold lower than those observed in failing human hearts. Conversely, inhibition of cardiac myocyte death in this murine model largely prevents the development of cardiac dilation and contractile dysfunction, the hallmarks of heart failure. To our knowledge, these data provide the first direct evidence that myocyte apoptosis may be a causal mechanism of heart failure, and they suggest that inhibition of this cell death process may constitute the basis for novel therapies.
\end{abstract}

J. Clin. Invest. 111:1497-1504 (2003). doi:10.1172/JCI200317664.

\section{Introduction}

Heart failure is a major cause of morbidity and mortality worldwide (1). Regardless of the initiating disease process, pathogenesis inevitably proceeds through a final common state termed dilated cardiomyopathy, in which heart chambers become markedly enlarged and contractile function deteriorates. Although abnormalities in cell membrane and cytoskeletal organization (2), adrenergic signaling (3), intracellular calcium handling $(4,5)$, and myocardial energetics (6) have been observed, the molecular and cellular mechanisms that mediate the pathogenesis of heart failure are poorly understood.

Recently, myocyte apoptosis has been noted in failing human hearts. While initial studies reported unrealistically high levels of cell death, probably due to methodological problems (7), later work has

Received for publication December 19, 2002, and accepted in revised form February 25, 2003.

Address correspondence to: Richard N. Kitsis, Departments of Medicine (Molecular Cardiology) and Cell Biology, Albert Einstein College of Medicine, 1300 Morris Park Avenue, Bronx, New York 10461, USA. Phone: (718) 430-2609; Fax: (718) 430-8989; E-mail: kitsis@aecom.yu.edu.

Conflict of interest: Robert C. Armstrong is an employee of Idun Pharmaceuticals. Richard N. Kitsis is a consultant to Idun Pharmaceuticals.

Nonstandard abbreviations used: FK-binding protein (FKBP); maximal rate of increase of left ventricular systolic pressure $(+\mathrm{dP} / \mathrm{dt})$; maximal rate of decrease of left ventricular systolic pressure $(-\mathrm{dP} / \mathrm{dt}) ; N-[(1,3-$ dimethylindole-2-carbonyl)valinyl]3-amino-4-oxo-5-fluoropentanoic acid (IDN 1965). consistently shown that approximately $80-250$ heart muscle cells per $10^{5}$ cardiac nuclei commit suicide at any given time in patients with late-stage dilated cardiomyopathy (8-10). In contrast, the base-line rate of apoptosis in healthy human hearts is only one to ten cardiac myocytes per $10^{5}$ nuclei. Whether the chronically elevated but extremely low levels of myocyte apoptosis observed in failing hearts play a causal role in the disease process remains a controversial issue with major therapeutic implications.

Apoptosis in all metazoan cells is mediated by caspases, a multigene family of cysteine proteases that hydrolyzes peptide bonds carboxyl to aspartic acid residues (11). Synthesized as zymogens, procaspase activation involves cleavage and the noncovalent reassociation of two p20 and two p10 fragments into an active holoenzyme. Once activated, caspases cut key cellular proteins, leading to the apoptotic demise of the cell. Caspases can be activated by at least two biochemical mechanisms. First, already activated upstream caspases cleave downstream procaspases. Second, the most upstream procaspases, such as procaspase-8, possess low but significant basal caspase activity and are thought to autoactivate in trans when forced into close proximity through interactions with other proteins (12).

We exploited this forced-proximity mechanism to create transgenic mice with cardiac-specific expression of a ligand-activated caspase. These mice provided a means to specifically manipulate the central death machinery and quantitatively modulate the induction 
of apoptosis in cardiac myocytes in vivo. Using this system, we discovered that very low levels of myocyte apoptosis, levels that are four- to tenfold lower than those seen in human heart failure, are, in fact, sufficient to cause a lethal, dilated cardiomyopathy. Moreover, inhibition of this cell death markedly inhibits the development of this syndrome. These experiments provide direct evidence that chronically elevated but low levels of apoptosis may play a causal role in the pathogenesis of heart failure.

\section{Methods}

Materials. Chemicals were purchased from SigmaAldrich (St. Louis, Missouri, USA) unless otherwise noted. C57BL/6 mice were supplied by Taconic (Germantown, New York, USA) and Charles River Laboratories (Wilmington, Massachusetts, USA). All animal experimental protocols were approved by the Institute for Animal Studies of the Albert Einstein College of Medicine.

Generation of FK-binding-protein-caspase-8 transgenic mice. To construct the transgene, we first subcloned the NotI-EcoRI fragment encoding the FK-bindingprotein-caspase-8 (FKBP-caspase-8) fusion protein (Figure 1a; gift of V.M. Dixit, Genentech Inc., South San Francisco, California, USA) (13) into pBluescript II KS+ (Stratagene, La Jolla, California, USA). We then subcloned the NotI blunted-HindIII fragment of this construct into the SalI blunted-HindIII sites of clone 26 , which contains $4.4 \mathrm{~kb}$ of mouse $\alpha$-cardiac myosin heavy chain $5^{\prime}$ flanking sequence (gift of J. Robbins, Children's Hospital, Cincinnati, Ohio, USA). The transgene construct was liberated from prokaryotic sequences by BamHI digestion and injected into the pronuclei of fertilized mouse eggs derived from an FVB/N-C57BL/6 mating. Transgenic progeny were subsequently backcrossed onto a C57BL/ 6 background. Animals derived from four to eight backcrosses were used for experiments. WT littermates were always used for controls. The C360A mutant transgene construct was made by PCR sitedirected mutagenesis (PfuTurbo DNA Polymerase; Stratagene) of codon TGT to GCT in the FKBP-caspase- 8 transgene construct described above. Then the entire construct was resequenced to exclude additional mutations.

Founders were identified by Southern analysis of EcoRI-digested tail DNA with a probe consisting of an EcoRI-XbaI genomic fragment of the mouse $\alpha$-cardiac myosin heavy chain gene spanning from the 5 ' flanking region to intron 1 . This probe identified a $3.6-\mathrm{kb}$ transgene fragment and a $2.5-\mathrm{kb}$ fragment of the endogenous $\alpha$-cardiac myosin heavy chain. Subsequent generations were screened by PCR of toe DNA (14) using a three-primer reaction that identified a 324-bp product from the transgene and a 506-bp product from the endogenous $\alpha$-cardiac myosin heavy chain gene. The forward primer was GACAGCAGATCACGATTCTC (corresponding to the $\alpha$-cardiac myosin heavy chain promoter). The reverse primers were TCCTTAGGCTTGCTCTTGC (corresponding to the myristoylation site of the transgene protein) and TTGCCACCATTGCACGTAC (corresponding to endogenous $\alpha$-cardiac myosin heavy chain sequences distal to those in the transgene construct).

Immunoblots. Mouse hearts were homogenized in 10 volumes of $50 \mathrm{mM} \mathrm{KCl}, 50 \mathrm{mM}$ PIPES (pH 7.4), 10 mM ethylene glycol-bis(2-aminoethylether)- $N, N, N^{\prime}, N^{\prime}$ tetraacetic acid, $2 \mathrm{mM} \mathrm{MgCl}_{2}$, and $1 \mathrm{mM} \mathrm{DTT}$ at $4^{\circ} \mathrm{C}$ with five 5-second pulses at 9,600 rpm, using an UltraTurrax T25 homogenizer (Jahnke and Kunkel, Staufen, Germany). Homogenates were then centrifuged at 2,200 $\mathrm{g}$ for 15 minutes, the supernatant was recovered, and centrifugation was repeated. The protein concentrations of the final supernatant were determined using the BCA Protein Assay Reagent (Pierce Chemical Co., Rockford, Illinois, USA). Twenty-microgram aliquots were resolved on $10 \%$ SDSPAGE gels and electroblotted onto $0.45-\mu \mathrm{m}$ nitrocellulose membranes (Bio-Rad Laboratories Inc., Hercules, California, USA). The blots were reacted with a 1:2,500 dilution of a rabbit polyclonal antibody against human caspase-8 (Idun Pharmaceuticals, San Diego, California, USA).

DNA ladder assay. A DNA ladder assay was performed as previously described (15).

TUNEL/actin costaining. A TUNEL/actin costaining assay was performed as previously described (15) with the following modifications pertaining to costaining for $\alpha$-sarcomeric actin. The TUNEL reaction was performed using a TACS 2 TdT DAB apoptosis detection kit (Trevigen Inc., Gaithersburg, Maryland, USA) according to the manufacturer's directions, except that the streptavidin used to detect the terminal deoxynucleotidyl transferase-ligated (TdT-ligated) biotinylated dNTPs was temporarily omitted. Staining for $\alpha$-sarcomeric actin was performed with a 1:30 dilution of rabbit polyclonal antibody (clone 5C5; Sigma-Aldrich). Following incubation with TRITCconjugated anti-rabbit secondary antibody (1:30 dilution) to detect the $\alpha$-sarcomeric actin antibody, Streptavidin-Orange-Green 488 (1:50 dilution; Jackson ImmunoResearch Laboratories Inc., West Grove, Pennsylvania, USA) was applied to detect ligated biotinylated dNTPs in apoptotic nuclei. Total nuclei on each section were visualized by staining with 500 $\mathrm{ng} / \mathrm{ml}$ bisbenzimide (Hoechst 33258). Sections were mounted on gridded coverslips (Bellco Glass Inc., Vineland, New Jersey, USA) and visualized by confocal light microscopy (Zeiss Axiophot; Carl Zeiss Inc., Thornwood, New York, USA).

For each section, the number of TUNEL-positive myocyte nuclei in the left ventricular free wall was manually counted. Only nuclei that were clearly located in cardiac myocytes were scored. The total number of nuclei in the left ventricular free wall was determined by automated counting of the bisbenzimide-positive signals using IPLab (Scanalytics Inc., 
Vienna, Virginia, USA). Five transverse sections spaced through the heart were analyzed for each animal. For controls and each of the three transgenic lines, three animals were studied per group.

Echocardiographic measurements. Echocardiographic studies (16) were performed with an HDI 5000cv ultrasonograph system (Advanced Technologies Laboratories, Andover, Massachusetts, USA). Mice were lightly anesthetized with methoxyflurane and placed in the supine position on a heating pad. A $10-\mathrm{MHz}$ transducer was applied to the left hemithorax, and two-dimensionally directed $\mathrm{M}$-mode images of the heart were recorded on videotape. Left ventricular dimensions and wall thicknesses were assessed at end diastole and end systole according to the guidelines of the American Society of Echocardiography. Data from three to six consecutive cardiac cycles were analyzed and averaged. Heart rate was determined from simultaneous electrocardiographic recordings. Left ventricular fractional shortening (LVFS) was calculated by the following formula: LVFS $(\%)=[($ LVEDD - LVESD $) /$ LVEDD $] \times 100$, where LVEDD indicates left ventricular end-diastolic dimension and LVESD indicates left ventricular endsystolic dimension. The data were analyzed by an observer blinded to genotype and/or treatment group. In vivo hemodynamic measurements. Left ventricular catheterization was performed under light methoxyflurane anesthesia with mice supine on a heating pad. A 1.4 F Millar catheter (Millar Instruments Inc., Houston, Texas, USA) was introduced into the right carotid artery and advanced to the left ventricle. Heart rate and left ventricular pressures were recorded under basal conditions and in response to $500 \mathrm{pg}$ isoproterenol i.v. Maximal rate of rise $(+\mathrm{dP} / \mathrm{dt})$ and fall $(-\mathrm{dP} / \mathrm{dt})$ of left ventricular systolic pressure was derived from the primary measurements. The data were analyzed by an observer blinded to genotype.

Statistical analysis. Results are presented as means \pm SEM. Statistical comparisons were performed using ANOVA and the Tukey multiple-comparison test, with differences considered significant at $P<0.05$. Kaplan-Meier survival analysis was used to assess all causes of mortality in the different groups.

\section{Results}

Generation of transgenic mice with inducible cardiac myocyte apoptosis. To explore whether cardiac myocyte apoptosis may be a mechanism of heart failure, we created transgenic mice in which heart muscle cell death could be activated at will. This was accomplished by the cardiac-specific expression of a fusion protein (Figure 1a) consisting of three modules of human FKBP-12 (pk mutant) attached to the p20 and p10 catalytic domains of human procaspase- 8 (13). This FKBP-caspase- 8 fusion protein would be predicted to be catalytically inactive unless forced into close proximity. Forced approximation can be stimulated by systemic administration to mice of FK1012H2, a small molecule that can simultaneously bind two FKBP modules and, thereby, induce the oligomerization of the transgene protein (17).

Southern blot analysis demonstrated the transgene in ten independent lines, two of which, lines 7 and 169 (Figure 1b), represent the highest and lowest expressers, respectively (Figure 1c). As a control for potential effects of overexpression, an additional transgenic line was generated that expresses comparable levels of the identical transgene protein except for a point mutation (C360A) that ablates caspase activity (Figure 1c). Three-week-old pups from each of these transgenic lines appeared grossly normal and exhibited no histological or echocardiographic abnormalities (not shown).

Effects of acute induction of apoptosis. To determine the effects of caspase activation and induction of cardiac myocyte apoptosis in these apparently normal animals, mice were injected with FK1012H2 or vehicle control. No gross or histological abnormalities developed following administration of vehicle to any of the animals or FK1012H2 to WT animals. In contrast, following a

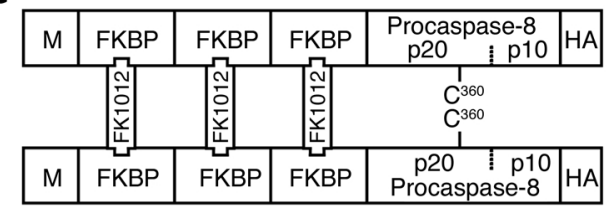

b

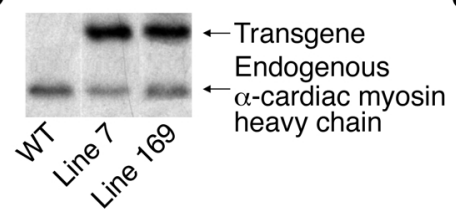

c

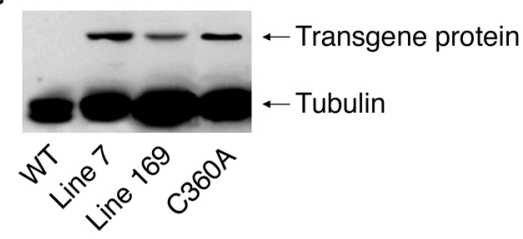

\section{Figure 1}

Expression of a conditionally active caspase- 8 allele in the hearts of transgenic mice. (a) Structure of the transgene protein and strategy for its activation. M, myristoylation signal; FKBP, human FKBP-12 (pk mutant); p20 and p10, 20- and 10-kDa domains, respectively, of human procaspase-8; HA, hemagglutinin tag; FK1012, dimerizer (see text). Cysteine 360, a residue essential for caspase activity, is shown. (b) Southern blot analysis of EcoRI-digested genomic DNA from WT mice and two of the ten transgenic lines generated. The probe, an EcoRI-Xbal mouse $\alpha$-cardiac myosin heavy chain genomic fragment, identified a 3.6-kb transgene fragment and a 2.5 -kb fragment of the endogenous $\alpha$-cardiac myosin heavy chain. (c) Immunoblot analysis using an antibody against human caspase-8, showing transgene protein expression in the hearts of the most highly expressing (line 7) and least highly expressing (line 169) lines at 3 weeks of age. Levels of the point-mutated transgene protein in the hearts of line C360A mice are similar to those of the catalytically active transgene protein in the hearts of line 7 mice. The transgene protein was not detectable in a survey of other organs, as expected with the cardiac myocyte-specific $\alpha$-cardiac myosin heavy chain promoter (not shown). The lower portion of the blot was reacted with an antibody against mouse tubulin as a loading control. 
FK1012H2 administration, all line $7(n>100)$ and line $169(n=25)$ transgenic mice died (Figure 2a). The time to death varied inversely with the dose of FK1012H2, ranging from 4 to 18 hours (Figure $2 \mathrm{~b}$ ). The lethal effect of FK1012H2 was dependent on caspase function, as the C360A transgenic line, which expresses comparable levels of the point-mutated, catalytically inactive caspase, was completely resistant to even the highest doses of this

$\mathbf{a}$
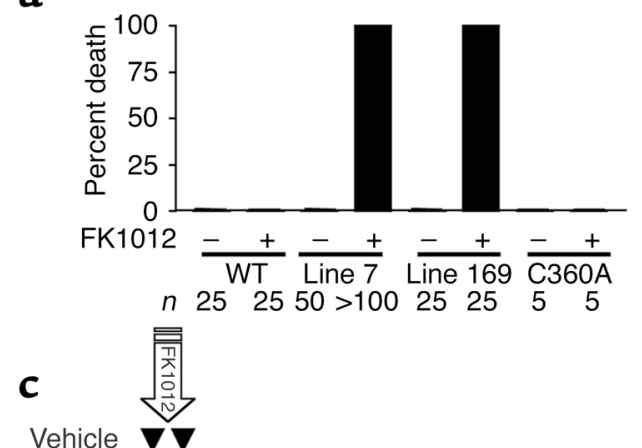

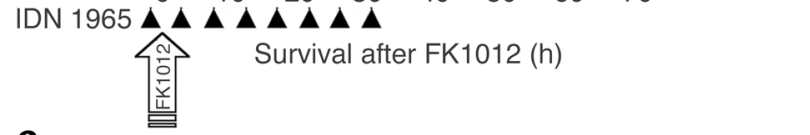

$\mathbf{e}$

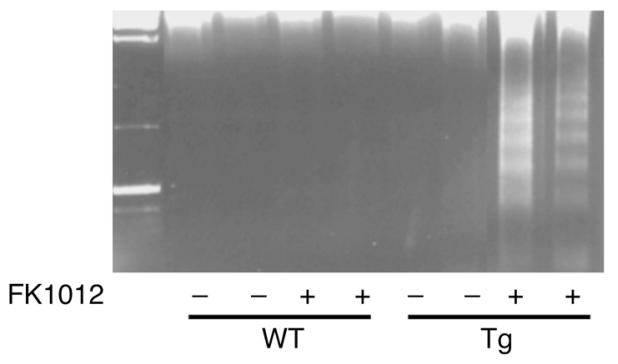

drug (Figure 2a). In addition, FK1012H2-induced death in line 7 mice was markedly delayed by coadministration of $N$-[(1,3-dimethylindole-2-carbonyl)valinyl]-3-amino4-oxo-5-fluoropentanoic acid (IDN 1965; Idun Pharmaceuticals), a dipeptide fluoromethylketone irreversible pseudosubstrate polycaspase inhibitor (Figure 2c). As expected, FK1012H2 triggered proteolytic cleavage of the transgene protein in the hearts of transgenic lines 7 b

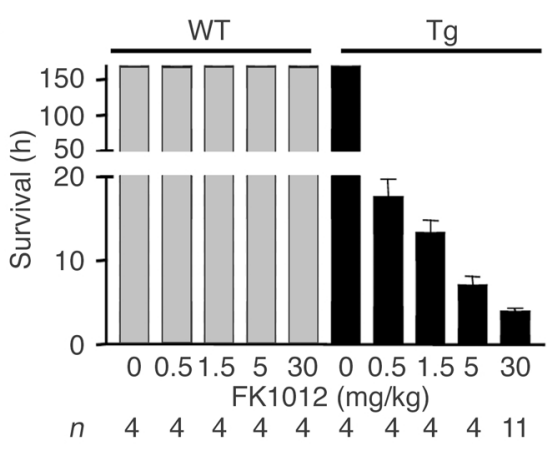

d

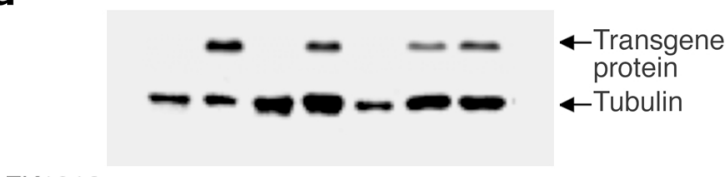

FK1012 f

Vehicle

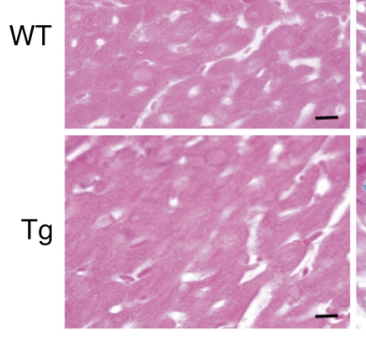

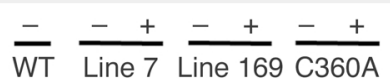

Figure 2

Activation of the caspase transgene in vivo results in massive cardiac myocyte apoptosis and death of the animals. (a) Mortality following intraperitoneal administration of vehicle alone (81.9\% polyethylene glycol 400, 9.1\% Tween-80, 9\% dimethylacetate) or FK1012H2 (30 mg/kg in the vehicle) to 3-week-old WT mice and transgenic line 7, 169, and C360A mice. Animals were observed for death for 7 days following FK1012H2 administration. (b) Inverse dose-dependence of the time to death following intraperitoneal administration of the indicated doses of FK1012 H2 to WT or transgenic line 7 (TG) mice. Animals were observed for death for 7 days following FK1012H2 administration, with no deaths occurring in the WT group. (c) Caspase inhibition delays time to death following transgene activation. Vehicle (0.9\% saline) or the polycaspase inhibitor IDN 1965 (12 mg/kg in vehicle) was administered intraperitoneally to line 7 transgenic mice 45 minutes before FK1012 H2 (30 mg/kg intraperitoneally) and every 4 hours thereafter as indicated by the triangles. Bars represent the survival times of individual animals that received vehicle or IDN 1965. Animals were observed for death until all had died. ${ }^{*} P<0.0001$. (d) Activation of the transgene caspase by FK1012 H2. Immunoblot of cardiac homogenates from WT and transgenic line 7, 169, and C360A mice treated 1.5 hours earlier with vehicle or FK1012 H2 (30 mg/kg intraperitoneally). Processing of procaspase- 8 is indicated by disappearance of the uncleaved moiety; cleavage fragments are not reliably detected in tissue homogenates, presumably because of rapid degradation. The lower portion of the blot was reacted with an antibody against mouse tubulin as a loading control. (e) Induction of cardiac apoptosis by FK1012H2. Genomic DNA from the hearts of WT and transgenic line 7 mice 1 hour after the administration of vehicle or FK1012H2 (30 mg/kg i.v.) was size-fractionated on an agarose gel containing ethidium bromide. (f) TUNEL analysis of FK1012H2-induced apoptosis. Paraffin-embedded sections of hearts from WT and line 7 transgenic mice 1 hour after administration of vehicle or FK1012H2 (30 mg/kg i.v.). TUNEL-positive cells were primarily myocytes, but additional unidentified cells were also present that may represent degenerating myocytes or infiltrating inflammatory cells due to magnitude and rapidity of the death. Bar, $20 \mu \mathrm{M}$. 
and 169 mice, consistent with caspase activation; in contrast, the catalytically inactive mutant protein in C360A hearts remained intact (Figure $2 \mathrm{~d}$ ). Analysis of cardiac DNA from line 7 transgenic mice treated with FK1012H2 revealed strong internucleosomal laddering consistent with apoptosis (Figure 2e). Similarly, TUNEL of heart sections demonstrated abundant DNA-strand breaks in line 7 transgenic mice that received FK1012H2 (Figure 2f). Echocardiography of these mice revealed marked increases in wall thickness, which, upon histological examination, were found to represent edema (not shown). Thus, activation of exogenous caspase- 8 in the heart results in massive cardiac myocyte apoptosis and death of the mouse.

Effects of chronically low levels of cardiac myocyte apoptosis. Our intention in generating the FKBP-caspase- 8 mice was to create a model in which the effects of low levels of myocyte apoptosis on cardiac structure and function could be evaluated. Clearly, the rapidity and magnitude of cell death following FK1012H2-induced caspase activation did not accurately model the very low levels of cardiac myocyte loss observed during heart failure in humans and rodents. Unexpectedly, however, even in the absence of FK1012H2, transgenic mice from the highly expressing transgenic line 7 were noted to exhibit increased mortality beginning at approximately 8-9 weeks of age (Figure 3a). In contrast, normal longevity was observed in the low-expressing transgenic line 169 mice (despite the rapid death exhibited by these animals when given FK1012H2) and in mice expressing the C360A mutant. We hypothesized that the decreased survival in line 7 mice resulted from cardiomyopathy due to low, but abnormal, levels of myocyte apoptosis. To test this hypothesis, cardiac structure and function were first

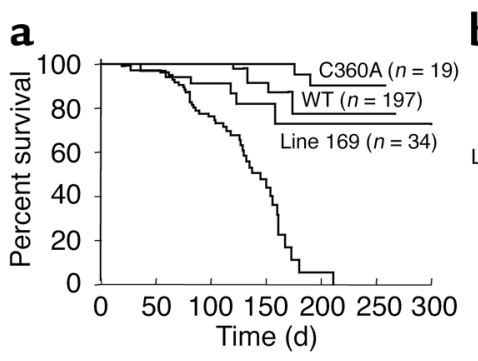

b
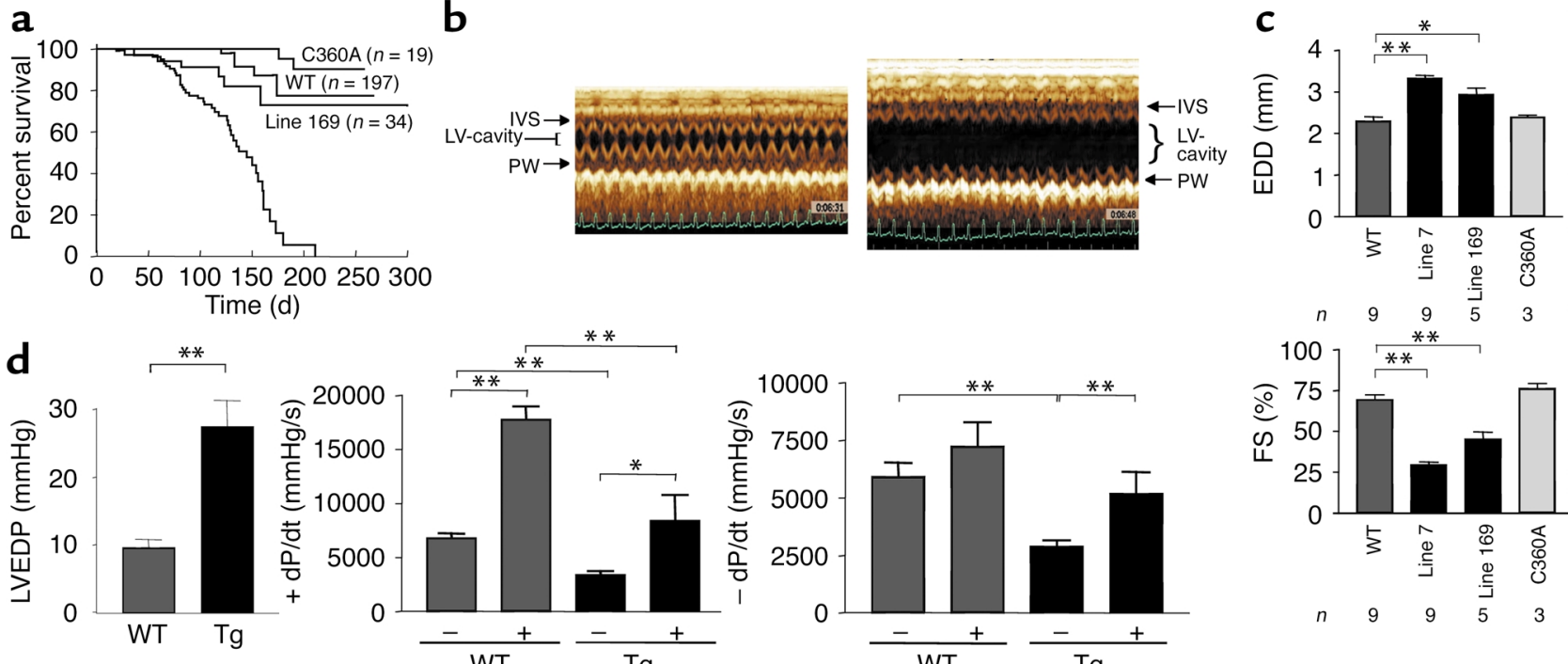

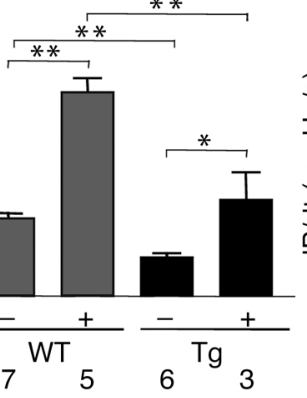

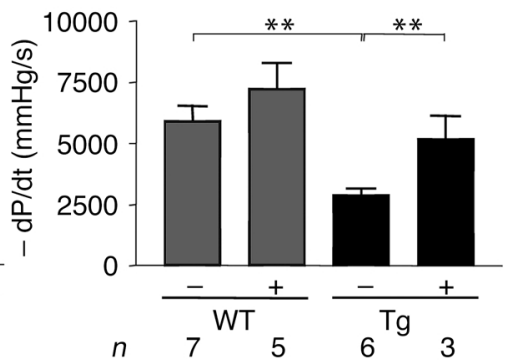

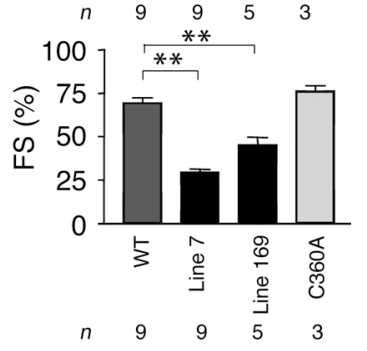

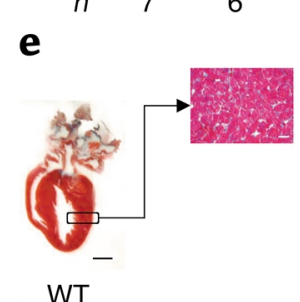

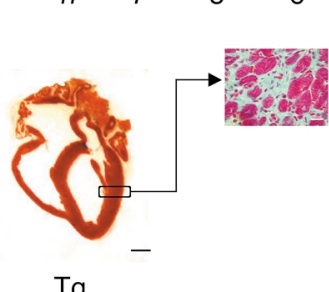

$\operatorname{Tg}$

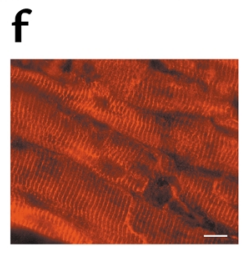

WT

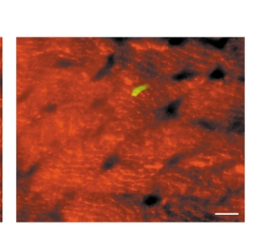

$\operatorname{Tg}$

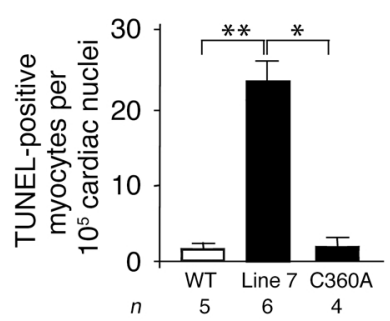

Figure 3

Very low levels of myocyte apoptosis are sufficient to cause a lethal, dilated cardiomyopathy. (a) Kaplan-Meier survival curve of WT mice, and transgenic line 7, 169, and C360A mice that have never been treated with FK1012H2. $P<0.0001$ for line 7 vs. WT, line C360A, or line 169. (b) Representative two-dimensionally directed M-mode echocardiograms through the interventricular septum (IVS) and left ventricular posterior wall (PW) from 9-week-old WT and transgenic line 7 mice in the absence of FK1012H2. The electrocardiogram is shown at the bottom of each echocardiogram. (c) Quantitation of M-mode echocardiographic parameters in conscious WT and transgenic line 7, 169, and C360A mice in the absence of FK1012H2. EDD, left ventricular end-diastolic dimension; FS, fractional shortening. ${ }^{*} P<0.01,{ }^{*} P<0.001$. (d) Left ventricular hemodynamics by cardiac catheterization in 9-week-old WT and transgenic line 7 mice under basal conditions or in response to isoproterenol (500 pg i.v.), in the absence of FK1012H2. LVEDP, left ventricular end-diastolic pressure. ${ }^{*} P<0.02,{ }^{*} P<0.002$. (e) Histological analysis of 9-week-old WT and transgenic line 7 mouse hearts in the absence of FK1012H2. Coronal sections stained with H\&E (bar, 1 $\mathrm{mm}$ ), and sections from the indicated area of the left ventricular free wall stained with Masson's trichrome (bar, $25 \mu \mathrm{m})$. (f) Apoptotic cardiac myocytes in WT and transgenic mice in the absence of FK1012H2. Left panels: Double staining for TUNEL (green) and desmin (red, to identify myocytes) in paraffin sections from the hearts of 9-week-old WT and line 7 transgenic mice in the absence of FK1012 H2. Bar, $10 \mu \mathrm{m}$. Right panel: Number of TUNEL-positive cardiac myocytes per $10^{5}$ nuclei in 9-week-old WT and transgenic line 7 and C360A mice in the absence of FK1012H2. ${ }^{*} P<0.002,{ }^{*} P<0.0003$. 
Table 1

Frequency of cardiac myocyte apoptosis in human heart failure vs. FKBP-caspase-8 mice

Species

$$
\begin{gathered}
\text { Apoptotic frequency } \\
\text { (myocytes per } 10^{5} \text { nuclei) }
\end{gathered}
$$

Controls

Dilated cardiomyopathy

Human heart failure

\begin{tabular}{lcc} 
Olivetti et al. (8) & 1 & 237 \\
Saraste et al. (9) & 11 & 119 \\
Guerra et al. (10) & 2 & $80-180$ \\
FKBP-caspase-8 mice & 1.6 & 23 \\
\hline
\end{tabular}

Apoptosis was assessed using the TUNEL assay.

evaluated at several time points. At 3 weeks of age, echocardiography and cardiac histology were normal (not shown). In contrast, by 9 weeks of age, line 7 transgenic mice showed left ventricular dilation and marked depression of fractional shortening (Figure 3, b and c). This was accompanied by histological myocyte dropout, interstitial fibrosis, thinning of the myocardium, and dilation of all four cardiac chambers (Figure 3e), indicative of dilated cardiomyopathy. Cardiac catheterization measurements showed elevated left ventricular end-diastolic pressures and depressed basal and isoproterenolstimulated $+\mathrm{dP} / \mathrm{dt}$ and $-\mathrm{dP} / \mathrm{dt}$ (Figure $3 \mathrm{~d}$ ), consistent with combined systolic and diastolic dysfunction. The lower-expressing line 169, which had normal longevity, exhibited an intermediate level of left ventricular dilation and contractile dysfunction (Figure $3 c$ ). In contrast, mice expressing the C360A mutant transgene had normal cardiac dimensions, function, and histology (Figure $3 c$ and data not shown). These data demonstrate that the FKBP-caspase-8 transgenic mice spontaneously develop a dilated cardiomyopathy between 3 and 9 weeks of age. This phenotype requires a catalytically active caspase, and the severity and mortality of this syndrome are related to the dose of the transgene protein.
To investigate the potential role of myocyte apoptosis in the dilated cardiomyopathy of the FKBP-caspase8 mice, TUNEL staining was performed on heart sections from 7.5- to 8.0-week-old animals that had never received FK1012H2 (Figure 3f). The frequency of spontaneous myocyte apoptosis in WT murine hearts was $1.59 \pm 0.7$ myocytes per $10^{5}$ cardiac nuclei, similar to that noted previously in healthy human hearts (8-10). Mice expressing the C360A inactive caspase exhibited similar basal rates. In contrast, the frequency of myocyte apoptosis in line 7 mice was $23.2 \pm 2.8$ myocytes per $10^{5}$ cardiac nuclei, 15 times higher than that in WT $(P<0.001)$. Despite being abnormally elevated, however, this frequency is still quite low. In fact, it is four to ten times lower than the most conservative estimates of myocyte death in failing human hearts (Table 1). These data demonstrate that induction of a very low level of myocyte apoptosis, lower than that observed in human heart failure, is sufficient to induce a lethal, dilated cardiomyopathy.

Prevention of cardiomyopathy by caspase inhibition. If the low levels of myocyte apoptosis in the FKBP-caspase- 8 transgenic mice really play a causal role in the resulting heart failure phenotype, then inhibition of this cell death should ameliorate the pathology. To test this, the polycaspase inhibitor IDN 1965 was administered to line 7 animals by continuous subcutaneous infusion via osmotic minipump. The infusion was started at 3.5-4.0 weeks of age, when the hearts were still normal, and continued to 7.5-8.0 weeks of age, when the cardiomyopathy is florid in untreated animals. As expected, caspase inhibition markedly reduced the rates of myocyte apoptosis (Figure 4a). Strikingly, inhibition of myocyte death was accompanied by complete abrogation of left ventricular dilation (Figure 4, b and c), dramatic improvement in left ventricular systolic function (Figure 4b), and lessening of histological features of cardiomyopathy such as fibrosis (Figure 4c). These

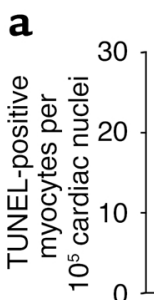

IDN 1965
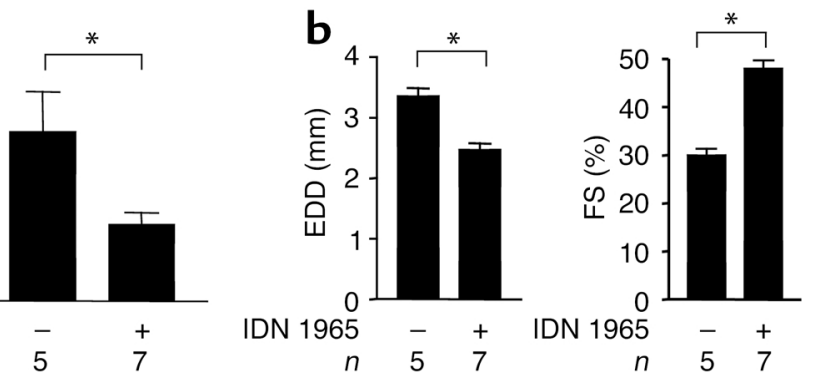

C

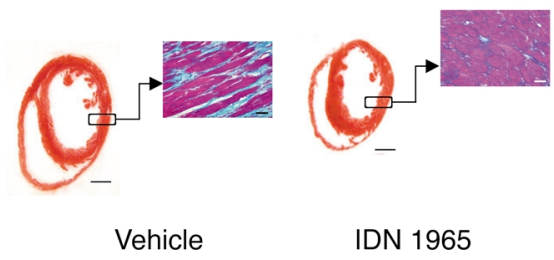

Figure 4

Abrogation of dilated cardiomyopathy by caspase inhibition. Vehicle or the polycaspase inhibitor IDN 1965 (12.5 $\mu \mathrm{g} / \mathrm{h})$ was administered to line 7 transgenic mice by continuous subcutaneous infusion using osmotic minipumps (model 1002; ALZET Corp., Cupertino, California, USA), beginning at 3.5-4.0 weeks of age, when cardiac dimensions, function, and histology are normal, and continuing until sacrifice at 7.5-8.0 weeks of age, when these transgenic mice uniformly exhibit a severe dilated cardiomyopathy. At 7.5-8.0 weeks of age, echocardiography, TUNEL, and histological examination of cardiac tissue were performed. (a) Number of TUNEL-positive cardiac myocytes per $10^{5}$ nuclei in vehicle- and IDN 1965-treated line 7 mice. ${ }^{*} P<0.03$. (b) M-mode echocardiographic parameters from vehicle- and IDN 1965-treated line 7 mice. ${ }^{*} P<0.0003$. (c) Coronal sections from vehicle- and IDN 1965-treated line 7 mice stained with H\&E (bar, 1 mm), and sections from the indicated area of the left ventricular free wall stained with Masson's trichrome (bar, $20 \mu \mathrm{m}$ ). 
data provide direct evidence that, in this model, myocyte apoptosis plays a critical role in the development of dilated cardiomyopathy.

\section{Discussion}

In this study, transgenic mice were generated with cardiac-restricted expression of a caspase- 8 fusion protein that could be activated by systemic administration of an exogenous ligand. Acute activation, as demonstrated by processing of the transgene protein, resulted in massive cardiac myocyte apoptosis and rapid organismal death. These effects were specific for the transgene protein, as they occurred in a ligand dose-dependent manner and were abrogated by caspase inhibitors or a point mutation that ablates caspase activity. Unexpectedly, however, even in the complete absence of activating ligand, the most highly expressing transgenic line exhibited very low, but abnormal, levels of cardiac myocyte apoptosis. This was accompanied by the gradual development of left ventricular dilation, systolic and diastolic dysfunction, and premature death, all consistent with a lethal, dilated cardiomyopathy. A second lower-expressing transgenic line manifested an intermediate phenotype, while a third line that expressed high levels of the transgene protein with an inactivating point mutation was normal. Attenuation of cardiac myocyte apoptosis by long-term caspase inhibition prevented the development of cardiomyopathy in the transgenic mice expressing high levels of the WT caspase-8 fusion protein. These data indicate that very low levels of cardiac myocyte apoptosis are sufficient to cause a lethal, dilated cardiomyopathy and that apoptosis is critical for disease pathogenesis in this model.

Low levels of cardiac myocyte death have long been postulated to be important in the pathogenesis of heart failure since the first descriptions of myocyte dropout in autopsy specimens. Despite this, the small magnitude of this cell loss at any given time point has raised significant doubts as to its importance in pathogenesis. Several previous studies exploring the relationship between cardiac myocyte apoptosis and heart failure have employed perturbations with potentially pleiotropic effects. These include drugs (18), genetic inactivation of an entire signaling pathway (19), and models with levels of cardiac myocyte apoptosis that were either equivocal (20) or far in excess of (19) those observed in heart failure. These features have precluded the establishment of an unambiguous relationship between cell death and disease pathogenesis.

In contrast, by directly manipulating one specific component of the central death machinery, the current study demonstrates a causal relationship between myocyte death and cardiomyopathy. Specifically, the data demonstrate, for the first time to our knowledge, that apoptotic rates of only 23 cardiac myocytes per $10^{5}$ nuclei are sufficient to induce a lethal, dilated cardiomyopathy. Although this frequency of cell death is quite low, it is still 15 -fold higher than that observed in the hearts of healthy, WT mice (one to two cardiac myocyte nuclei per $10^{5}$ nuclei). The most likely mechanism for the spontaneous low levels of cardiac myocyte apoptosis in the highly expressing line is low-level caspase activation due to spontaneous oligomerization of the transgene protein. This, in turn, was most likely facilitated by mass action, as evidenced by the more severe phenotype in the highly expressing line. Given the very low frequency of cardiac myocyte apoptosis, however, it is impossible to detect transgene protein oligomerization using biochemical approaches.

It is noteworthy that the level of cardiac myocyte apoptosis sufficient to induce cardiomyopathy in our transgenic mice is actually four- to tenfold lower than that measured in three independent studies of failing human hearts (8-10). Although caution must be exercised in making interspecies comparisons, the implication is that the low frequency of cardiac myocyte apoptosis in failing human hearts may also play an important role in heart failure pathogenesis.

The presence of spontaneous cardiomyopathy in the FKBP-caspase-8 mice, but not in controls expressing the mutated transgene protein, demonstrates the sufficiency of caspase activation and myocyte apoptosis to cause cardiomyopathy. Even if myocyte apoptosis is sufficient to cause cardiomyopathy, however, is it essential? Our experiments showing that inhibition of cardiac myocyte apoptosis results in the near-complete prevention of the cardiomyopathy demonstrate that cell death is indeed a critical factor in our model. In addition, a suggestion as to the general importance of myocyte death in heart failure is provided by other studies in our laboratory showing that inhibition of myocyte apoptosis rescues the peripartum cardiomyopathy in transgenic mice that overexpress Gaq in the heart (Y. Hayakawa and R.N. Kitsis, unpublished observations). To determine the universality of myocyte apoptosis as a pathogenic factor in heart failure, however, it will be necessary to test the effect of inhibition of apoptosis in the context of multiple other models.

An important caveat in the interpretation of our data involves the mechanism by which caspase activation leads to cardiomyopathy and, conversely, caspase inhibition prevents it. The most likely and parsimonious mechanism by which caspase activation contributes to cardiomyopathy is through cardiac myocyte death. This possibility is strongly supported by our data showing a tight link between the induction and inhibition of cardiac myocyte apoptosis and the development and prevention, respectively, of cardiomyopathy. It is possible, however, that caspases also affect cardiac structure and function independently of their modulation of apoptosis. One such mechanism could involve the processing of proinflammatory cytokines, although this is generally carried out by members of the caspase- 1 subfamily (21-23) and inflammation was not a significant feature of our model. Another potential mechanism could be 
the cleavage of sarcomeric proteins, as has been suggested for caspase-3 (24-26). The effects of caspase-mediated cleavage of contractile proteins on muscle function and long-term cellular viability remain to be determined.

In summary, we believe that this study provides the first direct evidence that chronic, low levels of cardiac myocyte apoptosis are a causal component in the pathogenesis of heart failure, and it raises the possibility that inhibition of this cell death may provide a novel target for treatments directed at this common and lethal disorder.

\section{Acknowledgments}

R.N. Kitsis was supported by grants from the NIH (R01 HL60665 and R01 HL61550) and by the Monique WeillCaulier Scholar Award. R.N. Kitsis is the Charles and Tamara Krasne Faculty Scholar in Cardiovascular Research of the Albert Einstein College of Medicine. D. Wencker was supported by the Glorney-Raisbeck Fellowship in Cardiovascular Diseases of the New York Academy of Medicine and an Individual National Research Service Award from the NIH. We are indebted to ARIAD Pharmaceuticals Inc. (Cambridge, Massachusetts, USA) for providing FK1012H2 (http://www.ariad.com/ regulationkits) and to Idun Pharmaceuticals for IDN 1965 and the caspase- 8 antibody.

1. Braunwald, E., and Bristow, M.R. 2000. Congestive heart failure: fifty years of progress. Circulation. 102:IV14-IV23.

2. Chien, K.R. 1999. Stress pathways and heart failure. Cell. 98:555-558. 3. Lefkowitz, R.J., Rockman, H.A., and Koch, W.J. 2000. Catecholamines, cardiac beta-adrenergic receptors, and heart failure. Circulation. 101:1634-1637.

4. Marks, A.R. 2002. Ryanodine receptors, FKBP12, and heart failure. Front. Biosci. 7:d970-d977.

5. Luo, W., et al. 1994. Targeted ablation of the phospholamban gene is associated with markedly enhanced myocardial contractility and loss of beta-agonist stimulation. Circ. Res. 75:401-409.

6. Taegtmeyer, H. 2002. Switching metabolic genes to build a better heart Circulation. 106:2043-2045.

7. Narula, J., et al. 1996. Apoptosis in myocytes in end-stage heart failure. N. Engl. J. Med. 335:1182-1189.
8. Olivetti, G., et al. 1997. Apoptosis in the failing human heart. N. Engl. J. Med. 336:1131-1141.

9. Saraste, A., et al. 1999. Cardiomyocyte apoptosis and progression of heart failure to transplantation. Eur. J. Clin. Invest. 29:380-386.

10. Guerra, S., et al. 1999. Myocyte death in the failing human heart is gender dependent. Circ. Res. 85:856-866.

11. Thornberry, N.A., and Lazebnik, Y. 1998. Caspases: enemies within. Science. 281:1312-1316.

12. Salvesen, G.S., and Dixit, V.M. 1999. Caspase activation: the inducedproximity model. Proc. Natl. Acad. Sci. U. S. A. 96:10964-10967.

13. Muzio, M., Stockwell, B.R., Stennicke, H.R., Salvesen, G.S., and Dixit, V.M. 1998. An induced proximity model for caspase-8 activation. J. Biol. Chem. 273:2926-2930.

14. Walter, C.A., Nasr-Schirf, D., and Luna, V.J. 1989. Identification of transgenic mice carrying the CAT gene with PCR amplification. Biotechniques. 7:1065-1070.

15. Bialik, S., et al. 1997. Myocyte apoptosis during acute myocardial infarction in the mouse localizes to hypoxic regions but occurs independently of p53. J. Clin. Invest. 100:1363-1372.

16. Chandra, M., et al. 2002. Cardioprotective effects of verapamil on myocardial structure and function in a murine model of chronic Trypanosoma cruzi infection (Brazil Strain): an echocardiographic study. Int. J. Parasitol. 32:207-215.

17. Spencer, D.M., Wandless, T.J., Schreiber, S.L., and Crabtree, G.R. 1993. Controlling signal transduction with synthetic ligands. Science. 262:1019-1024.

18. Li, Z., Bing, O.H., Long, X., Robinson, K.G., and Lakatta, E.G. 1997. Increased cardiomyocyte apoptosis during the transition to heart failure in the spontaneously hypertensive rat. Am. J. Physiol. 272:H2313-H2319.

19. Hirota, H., et al. 1999. Loss of a gp130 cardiac muscle cell survival pathway is a critical event in the onset of heart failure during biomechanical stress. Cell. 97:189-198.

20. Condorelli, G., et al. 2001. Heart-targeted overexpression of caspase3 in mice increases infarct size and depresses cardiac function. Proc. Natl. Acad. Sci. U. S. A. 98:9977-9982.

21. Li, P., et al. 1995. Mice deficient in IL-1 beta-converting enzyme are defective in production of mature IL-1 beta and resistant to endotoxic shock. Cell. 80:401-411.

22. Kuida, K., et al. 1995. Altered cytokine export and apoptosis in mice deficient in interleukin-1 beta converting enzyme. Science. 267:2000-2003.

23. Gu, Y., et al. 1997. Activation of interferon-gamma inducing factor mediated by interleukin-1beta converting enzyme. Science. 275:206-209.

24. Laugwitz, K.L., et al. 2001. Blocking caspase-activated apoptosis improves contractility in failing myocardium. Hum. Gene Ther. 12:2051-2063.

25. Moretti, A., et al. 2002. Essential myosin light chain as a target for caspase-3 in failing myocardium. Proc. Natl. Acad. Sci. U. S. A. 99:11860-11865.

26. Communal, C., et al. 2002. Functional consequences of caspase activation in cardiac myocytes. Proc. Natl. Acad. Sci. U. S. A. 99:6252-6256. 